\title{
Un paso en el fortalecimiento de la Atención Primaria de Salud
}

\author{
One step forward to the strengthening of the primary health \\ care
}

El desarrollo de la atención primaria de salud (APS) en Cuba comenzó, cuando aún no se le llamaba así a este espacio de la salud pública, con la creación del Servicio Médico Social Rural en 1960. Continuó en forma acelerada con el modelo del policlínico integral primero y el comunitario después, hasta que en los primeros años de la década de los 80 (siglo $x x$ ), fue concebido y comenzó a desarrollarse en forma experimental primero y extendida después, un nuevo modelo de APS.

Se basó en que un médico tendría la responsabilidad del cuidado de la salud de todos los integrantes de la familia.

Esto exigió reformar los planes de estudio, que graduarían un médico general capaz de asumir la atención de toda la población, en lugar de aquellos que se venían graduando en los tres programas verticales orientados a niños, embarazadas y adultos.

Estos profesionales estarían preparados con conocimientos básicos de epidemiología, higiene ambiental y otros elementos que le permitieran desempeñarse a nivel primario en las tareas generales de las funciones de promoción de salud y prevención de enfermedades.

Completaba la idea esencial del nuevo modelo de APS, llevar a los médicos a trabajar y residir al lugar donde vivía la población que atendía, junto con él trabajaría también una enfermera en funciones asistenciales y sanitarias.

Muchos problemas debieron ser resueltos para implantar y alcanzar un elevado desarrollo de este modelo. Romper con lo establecido es siempre difícil y los opositores fueron numerosos e importantes.

Pero el modelo se abrió paso, con gran satisfacción de la población, con éxito expresado en mejoría de indicadores de salud, en consolidar un servicio de total cobertura, con alta equidad y calidad satisfactoria. Fue un logro notable que contuvo los embates del "período especial", con alta eficacia. 


\section{Revista Cubana de Salud Pública. 2011;37(3):193-194}

La fortaleza del modelo de APS cubano está basada en un médico que se desempeña como médico de familia y que cursando el plan de estudios apropiado, se convierte en especialista en medicina general integral (MGI). El trabajo desde el consultorio del médico y la enfermera comienza a debilitarse cuando se aprueba la segunda especialidad para los que ya eran especialistas de primer grado en MGI.

La resistencia inicial a dedicarse a MGI había cedido bastante. La práctica mostraba ya resultados muy positivos de realización personal de los especialistas en MGI. Al abrirse una opción para regresar a los viejos deseos de otras especialidades, pocos primero y muchos después, abandonaron la MGI y el consultorio. Esto alcanzó al grupo de los más destacados, que habían formado el contingente de vanguardia.

A los fundadores y primeros destacados, hoy no se les encuentra en aquellas posiciones, en la base, donde se hacen realidad los principios de la salud pública revolucionaria cubana.

Si lo anterior es cierto, para fortalecer la APS, propósito declarado por la alta dirección del Sistema Nacional de Salud, lo primero deberá ser estimular la permanencia de los especialistas en MGI en el espacio de la APS y limitar la opción referida, que dio inicio a la pérdida de grupos importantes de especialistas en MGI hacia otras tareas fuera de la APS.

Esta limitación será regulada, admitiendo el acceso a la segunda especialidad al número que se demuestre son necesarios al servicio, en la propia APS o fuera de ella.

El objetivo concreto será estabilizar en sus consultorios o policlínicos la cifra numerosa de médicos imprescindibles en estos servicios.

Esto no será lo único que deba hacerse. Una política general que estabilice y fortalezca el modelo de APS tendrá que atender y resolver numerosos escollos. Hacer esto es lo más importante hoy para la salud de nuestro pueblo. 